\title{
A MONOLITHIC FLUID-STRUCTURE INTERACTION METHOD, APPLICATION TO A PISTON PROBLEM
}

\author{
Felix Ischinger $^{1}$, Martijn Anthonissen ${ }^{2}$, and Barry Koren ${ }^{2}$ \\ ${ }^{1}$ Eindhoven University of Technology \\ e-mail: f.ischinger@student.tue.nl \\ ${ }^{2}$ Eindhoven University of Technology \\ Department of Mathematics and Computer Science \\ P.O. Box 513, 5600 MB Eindhoven \\ The Netherlands \\ e-mail: \{b.koren, m.j.h.anthonissen\}@ $@$ tue.nl
}

Keywords: fluid-structure interaction, monolithic approach, Euler equations of gas dynamics, acoustic equations, mass-spring system, Discontinuous Galerkin, immersed boundary method, Lyapunov stability

\begin{abstract}
A monolithic FSI method is presented. A standard piston problem is considered as test case. The piston problem's fluid domain is represented by a closed tube filled with air. One end of the fluid tube is formed by a piston connected to a spring. We use the Euler equations of gas dynamics as well as a linear simplification of these, the acoustic equations, to model the gas dynamics in the tube. A Discontinuous Galerkin method is applied to discretize the fluidflow equations, together with an immersed-boundary method to account for the moving piston. A monolithic formulation of the coupled system is derived and analyzed. It is proven that the semidiscrete formulation is stable, if two correction terms are used at the coupling interface. We use Lyapunov functions to prove stability of the semi-discrete monolithic formulation. Further, different time-integration methods are considered, analyzed and tested. The numerical results are very accurate; they correspond very well to analytical approximations. The theoretical prediction of the eigenfrequency can be reproduced very accurately. Moreover, the amplitude of the spring oscillation is conserved very well.
\end{abstract}




\section{INTRODUCTION}

The computer simulation of Fluid-Structure Interaction (FSI) has become a major research topic over the last two decades and is of interest to many applications. There are two main FSI approaches, the partitioned and the monolithic approach. The idea of the partitioned approach is to calculate the fluid flow and the structural movement separately. This has the advantage that already existing and specialized simulation tools can be used to calculate the dynamics of each system. The solvers are then applied alternatingly in time. The boundary conditions for one system are given by the state of the other system. Therefore, there is a time lag between the boundary conditions and the system. To avoid the time lag, monolithic methods are to be preferred over partitioned methods. In monolithic methods, the fluid and the structural part are evolved simultaneously in time. The challenge for monolithic methods lies in the correct mathematical formulation of the system. Both systems and the coupling between them have to be formulated in a single expression.

Another challenge in FSI computations is the numerical tracking of moving boundaries. In the Arbitrary-Lagrangian-Eulerian (ALE) approach, the computational grid is adapted at every time step to fit the geometry of the modeled problem. This may become computationally expensive. In contrast, there are Immersed Boundary (IB) methods, introduced by Peskin [1]. Over the last decades a lot of modifications were proposed for IB methods. The main idea is to use a non-deforming grid, which does not necessarily fit with the model geometry. IB methods are very well suited for complex geometries and large deformations. Furthermore, using an IB method can save a lot of computational time, because the computational grid does not have to be adapted at every time step. In this work, we use an IB method.

As a fluid-structure interaction problem we consider the piston problem from [2]. The model consists of a closed tube filled with air. The right end of the tube is closed by a piston that is attached to a spring and hence is movable (Figure 1). The pressure outside the tube is set constant to $p_{0}>0$. The left end of the tube is at $x_{\mathrm{LB}} \in \mathbb{R}$ and the piston wall is at position $x_{\text {wall }} \in \mathbb{R}$. The equilibrium position of the spring is denoted as $l_{0} \in \mathbb{R}$. The velocity of the piston is $\dot{x}_{\text {wall }}=u_{\text {wall }} \in \mathbb{R}$.

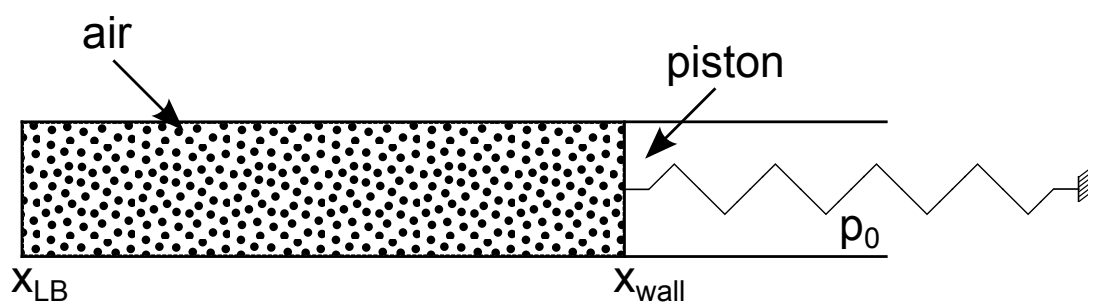

Figure 1: Sketch of the piston problem.

Some publications addressed the piston problem with an ALE approach. In [2] and [3] partitioned approaches are introduced. A first order accurate monolithic implicit scheme is proposed in [4]. Further work is presented in [5], [6] and [7]. In these publications, a timediscontinuous Galerkin method is used for the discretization of the fluid equations. This is related to an ALE approach. As mentioned, we will address the piston problem with an IB method.

The structural part of the FSI problem, the spring and the piston, is described by Newton's law. Two forces act on the piston, a restoring force $F_{r}$ which is determined by Hooke's law and 
a force $F_{p}$ resulting from the pressure difference over the piston, i.e.

$$
m \ddot{x}_{\text {wall }}(t)=F_{p}-F_{r}=A\left(p_{\text {wall }}-p_{0}\right)-k\left(x_{\text {wall }}-l_{0}\right),
$$

with $m$ the mass of the piston, $A$ the cross sectional area of the piston, $p_{\text {wall }}$ the pressure in the fluid at the left of the moving wall and $k$ the stiffness of the spring. (1) can be rewritten into a system of first order ordinary differential equations:

$$
\frac{d}{d t}\left(\begin{array}{l}
x_{\text {wall }} \\
u_{\text {wall }}
\end{array}\right)=\left(\begin{array}{cc}
0 & 1 \\
-\frac{k}{m} & 0
\end{array}\right)\left(\begin{array}{l}
x_{\text {wall }} \\
u_{\text {wall }}
\end{array}\right)+\left(\begin{array}{c}
0 \\
\frac{k}{m} l_{0}+\frac{A}{m}\left(p_{\text {wall }}-p_{0}\right)
\end{array}\right) .
$$

The air flow in the tube is modeled with a strictly hyperbolic system of conservation laws. We use the Euler equations and a linear simplification, the acoustic equations. The Euler equations describe the conservation of mass, momentum and energy of the gas. In one spatial dimension they read

$$
\frac{\partial}{\partial t}\left(\begin{array}{c}
\rho \\
\rho u \\
E
\end{array}\right)+\frac{\partial}{\partial x}\left(\begin{array}{c}
\rho u \\
\rho u^{2}+p \\
u(E+p)
\end{array}\right)=\left(\begin{array}{l}
0 \\
0 \\
0
\end{array}\right)
$$

$\rho$ denotes the density, $u$ denotes the velocity in $x$-direction and $E$ is the total energy per unit volume. $p$ is the pressure. An equation of state is necessary to close the system. For an ideal gas this is

$$
p=\left(E-\frac{1}{2} u^{2} \rho\right)(\gamma-1)
$$

with the ratio of specific heats $\gamma=1.4$. The Euler equations can also be written in quasi-linear form:

with the Jacobian of the flux $\boldsymbol{F}$

$$
\frac{\partial}{\partial t} \boldsymbol{u}+\boldsymbol{F}(\boldsymbol{u}) \frac{\partial}{\partial x} \boldsymbol{u}=\mathbf{0}
$$

$$
\boldsymbol{F}(\boldsymbol{u})=\frac{d \boldsymbol{f}(\boldsymbol{u})}{d \boldsymbol{u}}=\left(\begin{array}{ccc}
0 & 1 & 0 \\
-\frac{1}{2}(\gamma-1) u^{2} & (3-\gamma) u & \gamma-1 \\
-\frac{\gamma \rho u E}{\rho^{2}}+(\gamma-1)(u)^{3} & \frac{\gamma E}{\rho}-\frac{3}{2}(\gamma-1) u^{2} & \gamma u
\end{array}\right)
$$

The acoustic equations are a linear simplification of the Euler equations. In fluids, acoustics is defined as small, isentropic perturbations. Therefore, perturbations $\tilde{\rho}$ around a constant density $\bar{\rho}$ (we choose $\bar{\rho}=1.3 \mathrm{~kg} / \mathrm{m}^{3}$ ) and perturbations $\tilde{u}$ around a constant velocity $\bar{u}$ are considered. The gas is assumed to be at rest $(\bar{u}=0 \mathrm{~m} / \mathrm{s})$. This gives the expressions $\rho=\tilde{\rho}+\bar{\rho}$ and $u=\tilde{u}$.

The isentropic speed of sound is given by

$$
c=\sqrt{\left.\left(\frac{\partial p}{\partial \rho}\right)\right|_{s}}
$$

the square root of the derivative of the pressure with respect to the density at constant entropy $s$.

Substituting the expressions for density and velocity, as well as the isentropic speed of sound into the Euler equations and neglecting higher order terms gives

$$
\frac{\partial}{\partial t}\left(\begin{array}{c}
\rho \\
\bar{\rho} u
\end{array}\right)+\left(\begin{array}{cc}
0 & 1 \\
c^{2} & 0
\end{array}\right) \frac{\partial}{\partial x}\left(\begin{array}{c}
\rho \\
\bar{\rho} u
\end{array}\right)=\left(\begin{array}{l}
0 \\
0
\end{array}\right)
$$


The quasi-linear formulation can also be transformed into characteristic variables $\boldsymbol{\xi}$ with the help of diagonalization matrices $\boldsymbol{R}_{f}$ :

$$
\begin{aligned}
\boldsymbol{R}_{f}^{-1} \frac{\partial}{\partial t} \boldsymbol{u} & +\boldsymbol{R}_{f}^{-1} \boldsymbol{F} \boldsymbol{R}_{f} \boldsymbol{R}_{f}^{-1} \frac{\partial}{\partial x} \boldsymbol{u}=\mathbf{0} \\
\Leftrightarrow \quad \frac{\partial}{\partial t} \boldsymbol{\xi}+\boldsymbol{\Lambda} \frac{\partial}{\partial x} \boldsymbol{\xi} & =\mathbf{0} .
\end{aligned}
$$

The diagonalization matrix for the Euler equations is denoted as $\boldsymbol{R}_{f \text {,Euler }}$ and for the acoustic equations as $\boldsymbol{R}_{f, \text { ac }}$. The matrix $\boldsymbol{\Lambda}$ contains the eigenvalues $\lambda_{j}$ of the Jacobian matrix $\boldsymbol{F}$. The characteristic variables and the diagonalization matrices will appear later on for the numerical flux calculation and the stability analysis.

In [4] an analytical approach is introduced to predict the eigenfrequency of the piston problem when the acoustic equations are used. In contrast to the referred work, we do not neglect the cross sectional area $A$ of the piston and get the equation

$$
-m \omega^{2}+k+A \bar{\rho} \omega c \frac{1}{\tan \left(\frac{\omega}{c} L\right)}=0 .
$$

$\omega$ describes the eigenfrequency of the coupled piston system. $L$ is the mean length of the tube and defined as $L=l_{0}-x_{\mathrm{LB}}$. (11) can now be made dimensionless. We introduce the three dimensionless numbers

$$
X=\frac{\bar{\rho} A L}{m}, \quad Y=\sqrt{\frac{k}{m}} \frac{L}{c}, \quad Z=\frac{\omega L}{c} .
$$

(11) then reads

$$
-Z^{2}+Y^{2}+X Z \frac{1}{\tan (Z)}=0
$$

$X$ describes the mass ratios between the mass of the air in the tube $(\bar{\rho} A L)$ and the mass of the piston $(m)$. If the mass of the piston dominates the mass of the air inside the fluid tube, $X$ becomes small and can eventually be assumed to be zero. (13) can then be rewritten into an explicit formulation for $\omega$ :

$$
\omega^{2}=\frac{k}{m}
$$

This is just the eigenfrequency of a simple mass-spring system. We conclude that if the piston mass is dominating the mass of the air, then the eigenfrequency of the system is only determined by the spring and the fluid tube does not have any influence. Therefore, it could be reasonable for small values of $X$ to make the FSI coupling only in one direction, from the spring onto the fluid. In this case it is assumed that the fluid does not have any influence on the spring.

We choose the parameters for our test problem as $m=0.8 \mathrm{~kg}, k=0.8 \cdot 100 \mathrm{~kg} / \mathrm{s}^{2}, c=$ $328.17 \mathrm{~m} / \mathrm{s}, L=1 \mathrm{~m}$ and $A=1 \mathrm{~m}^{2}$. This results in an angular frequency of $\omega \approx 341.60681 / \mathrm{s}$, respectively an eigenfrequency of $f=\frac{\omega}{2 \pi} \approx 54.36841 / \mathrm{s}$. Further, we get $X=1.625$, thus a strong interplay between the fluid and the structure. Therefore a monolithic coupling is advisable.

\section{NUMERICAL SCHEME}

\subsection{Notation}

First we need to introduce some notation. For the piston problem, it is necessary to define some special notation. Two domains are defined. The first one is called the enclosing domain 


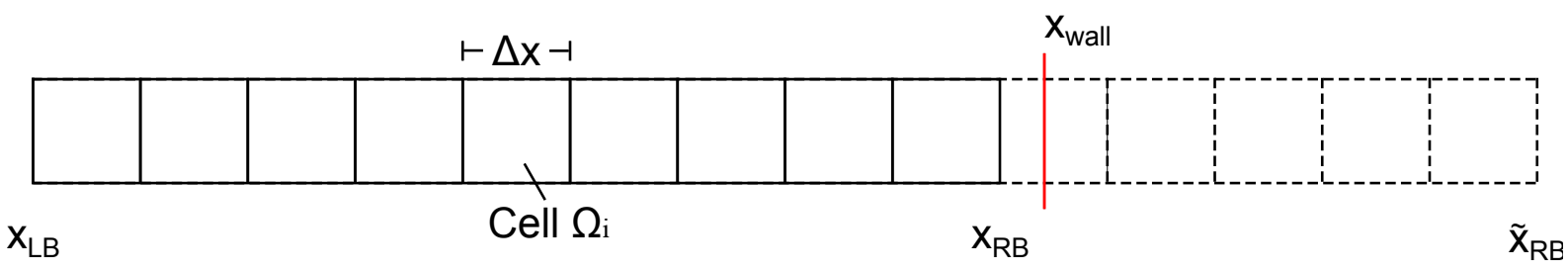

Figure 2: Sketch of the two domains. The computational domain $\Omega$ is drawn with solid lines. The enclosing domain $\Omega_{\text {tot }}$ is defined by all cells, indicated by the solid and dashed lines together.

$\Omega_{\text {total }}$ and represents the largest possible domain for the fluid. The second one is called $\Omega$ and it is a subset of the enclosing domain $\Omega \subseteq \Omega_{\text {total }}$. $\Omega$ is the computational domain. The left boundary of $\Omega_{\text {total }}$ and $\Omega$ is always the same as this end is immovable. It has the coordinate $x_{\mathrm{LB}}$. The right boundary of the enclosing domain $\Omega_{\text {total }}$ is also fixed. It has the coordinate $\tilde{x}_{\mathrm{RB}}$. It is required that the right boundary of $\Omega$ is always within the enclosing domain $\Omega_{\text {total }}$, that is $x_{\text {wall }} \leq \tilde{x}_{\mathrm{RB}}$. The right boundary of the computational domain is called $x_{\mathrm{RB}}$.

For the numerical discretization the enclosing domain $\Omega_{\text {total }}$ is split into $N_{\text {total }} \in \mathbb{N}$ cells $\Omega_{i}$ with equal lengths $\Delta x$. Therefore, the spatial discretization size becomes $\Delta x:=\frac{\tilde{x}_{\mathrm{RB}}-x_{\mathrm{LB}}}{N_{\mathrm{to}}}$. The boundaries of a cell $i$ have the coordinates $x_{i, \mathrm{~L}}$ for the left cell boundary and $x_{i, \mathrm{R}}$ for the right one. The number of cells in the computational domain is $N \leq N_{\text {total }}$.

The $i$-th cell is called $\Omega_{i}$. Every cell contains $p+1$ integration points $x_{i, k}$ where $i=1, \ldots, N$ is the index of the cell number and $k=1, \ldots, p+1$ indicates the integration point on which the solution of the Discontinuous Galerkin method is represented. $p \in \mathbb{N}_{0}$ is the polynomial degree of the solution. A Discontinuous Galerkin method with $p=0$ may be interpreted as a Finite-Volume method.

\subsection{Discontinuous Galerkin method}

The general expression for the Euler and the acoustic equations is

$$
\frac{\partial}{\partial t} \boldsymbol{u}(x, t)+\frac{\partial}{\partial x} \boldsymbol{f}(\boldsymbol{u}(x, t))=\mathbf{0} .
$$

The numerical solution of the DG method to approximate $\boldsymbol{u}(x, t)$ consists of $N$ polynomials of order $p$ for each conserved variable, referred to as $\boldsymbol{U}_{i}(x, t) \in\left(\mathbb{P}^{p}\left(\Omega_{i}\right)\right)^{d}$ with $i$ indicating the respective cell $\Omega_{i}$ and $\mathbb{P}^{p}\left(\Omega_{i}\right)$ the space of polynomials of order $p$ on $\Omega_{i}$. For the Euler equations, a system of three equations, $\boldsymbol{U}_{i}(x, t)$ is a vector of three polynomials $U_{i, j}(x, t)$, representing the conserved variables $\rho, \rho u$ and $E$. At each cell interface the solution is not unique but defined twice for each variable, because the cells are closed intervals. A detailed derivation and analysis of the Discontinuous Galerkin method can be found in [8].

We are using the nodal Discontinuous Galerkin approach. This means that the solution for one variable $j$ on one cell $i$ has the structure

$$
U_{i, j}(x, t)=\sum_{k=1}^{p+1} U_{i, j}\left(x_{k}, t\right) l_{k}(x)=\sum_{k=1}^{p+1} U_{i, j, k} l_{k}(x) .
$$

The Legendre-Gauß-Lobatto points are used as integration points $x_{i, k}$. They have the advantage that they always lie on the boundaries of the cells which will be helpful for the flux calculations. $l_{k}(x)$ are the Lagrange polynomials defined on cell $\Omega_{i}$. They are defined with the help of the 
integration points:

$$
l_{k}(x)=\prod_{\substack{m=1 \\ m \neq k}}^{p+1} \frac{x-x_{i, m}}{x_{i, k}-x_{i, m}}, \quad \text { for } x \in \Omega_{i} .
$$

$U_{i, j, k}$ are the integration coefficients and can be written more compactly in the vector $\boldsymbol{U} \in$ $\mathbb{R}^{N d(p+1)}$. This vector contains values for every integration point, every unknown variable and every cell. In total, the solution vector of the nodal DG method looks as follows:

$$
\boldsymbol{U}=\left(\begin{array}{c}
\boldsymbol{U}_{1} \\
\vdots \\
\boldsymbol{U}_{N}
\end{array}\right)=\left(\begin{array}{c}
\boldsymbol{U}_{1,1} \\
\vdots \\
\boldsymbol{U}_{1, d} \\
\vdots \\
\vdots \\
\boldsymbol{U}_{N, d}
\end{array}\right)=\left(\begin{array}{c}
U_{1,1,1} \\
\vdots \\
U_{1,1, p+1} \\
\vdots \\
\vdots \\
U_{N, d, p+1}
\end{array}\right)
$$

For the Discontinuous Galerkin discretization, we replace the exact solution in (15) by the nodal approach, multiply with test functions from $\mathbb{P}^{p}\left(\Omega_{i}\right)$ and integrate over cell $\Omega_{i}$. The flux term is simply approximated by

$$
\boldsymbol{f}\left(\boldsymbol{U}_{i}\right)_{j} \approx \sum_{k=1}^{p+1} \boldsymbol{f}\left(U_{i, j, k}(t)\right)_{j} l_{k}(x)=: \sum_{k=1}^{p+1} F_{i, j, k}(t) l_{k}(x) .
$$

Using integration by parts, we get

$$
\begin{gathered}
\int_{\Omega_{i}} \frac{\partial}{\partial t}\left(\sum_{k=1}^{p+1} U_{i, j, k}(t) l_{k}(x)\right) l_{m}(x) d \Omega_{i}-\int_{\Omega_{i}}\left(\sum_{k=1}^{p+1} F_{i, j, k}(t) l_{k}(x)\right) \frac{\partial}{\partial x} l_{m}(x) d \Omega_{i} \\
=\delta_{1, m}\left[g_{j}\left(\boldsymbol{U}_{i-1}, \boldsymbol{U}_{i}\right)\right]+\delta_{p+1, m}\left[-g_{j}\left(\boldsymbol{U}_{i-1}, \boldsymbol{U}_{i}\right)\right], \quad m=1, \ldots, p+1 .
\end{gathered}
$$

The flux at the right-hand side, that is evaluated at the boundary of the cell, is approximated with a numerical flux $g_{j}$, known from the Finite-Volume methods. As the Legendre-Gauss-Lobatto points are used, the cell boundaries are interpolation points. Therefore, as $l_{i}\left(x_{j}\right)=\delta_{i j}$, the value at the cell interface is exclusively defined by the outer interpolation points. $\delta_{i j}$ denotes the Kronecker delta. We use a flux-splitting approach to calculate the numerical fluxes:

$$
g_{j}\left(\boldsymbol{U}_{i-1}, \boldsymbol{U}_{i}\right)=\left(\boldsymbol{F}^{+} \boldsymbol{U}_{i-1}+\boldsymbol{F}^{-} \boldsymbol{U}_{i}\right)_{j} .
$$

The positive and negative fluxes are defined as

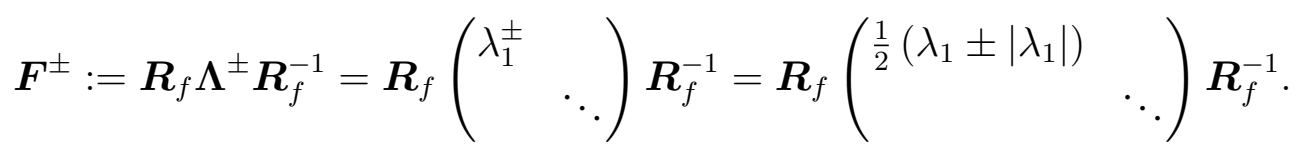

(20) is called the weak formulation of the DG method. After one more integration by parts, using the assumption that the values only depend on values from inside the cell, we get to the strong formulation of the DG scheme:

$$
\begin{aligned}
\int_{\Omega_{i}} \frac{\partial}{\partial t}\left(\sum_{k=1}^{p+1} U_{i, j, k} l_{k}(x)\right) & l_{m}(x)+\left(\frac{\partial}{\partial x} \sum_{k=1}^{p+1} F_{i, j, k} l_{k}(x)\right) l_{m}(x) d \Omega_{i} \\
= & \delta_{1, m}\left(-F_{i, j, 1}+g_{j}\left(\boldsymbol{U}_{i-1}, \boldsymbol{U}_{i}\right)\right)+\delta_{p+1, m}\left[F_{i, j, p+1}-g_{j}\left(\boldsymbol{U}_{i}, \boldsymbol{U}_{i+1}\right)\right] .
\end{aligned}
$$


In the remainder of this work we consider the strong formulation only. Next, the integrals will be projected on the reference cell $I=[-1,1]$ and we call the reference variable $r \in I$. The interpolation points on $I$ are called $r_{k}$. The following mapping from the reference variable $r$ to the actual variable $x$ is used:

$$
x(r)=x_{i, \mathrm{~L}}+\frac{1+r}{2} \Delta x, \quad-1 \leq r \leq 1, \quad \text { for } x \in \Omega_{i} .
$$

The transformation between $r$ and $x$ for some function $f$ under the integral is

$$
\int_{x_{i, \mathrm{~L}}}^{x_{i, \mathrm{R}}} f(x) d x=\int_{-1}^{1} f(x(r)) \frac{d x(r)}{d r} d r=\frac{\Delta x}{2} \int_{-1}^{1} f(r) d r .
$$

The transformation introduces a scaling factor $\frac{d x(r)}{d r}$ with $x(r)$ from (24). For the second integral, this scaling factor is compensated by the change of the derivative $\frac{d}{d x}$ to $\frac{d}{d r}$. The transformation becomes especially useful if the cells do not have the same size.

The integration of the multiplied Lagrange polynomials can be summarized in a mass matrix $\mathcal{M}$ with entries

$$
\mathcal{M}_{p q}=\int_{-1}^{1} l_{p}(x(r)) l_{q}(x(r)) d r=\int_{-1}^{1} \prod_{\substack{m=1 \\ m \neq p}}^{p+1} \frac{r-r_{m}}{r_{p}-r_{m}} \prod_{\substack{m=1 \\ m \neq p}}^{p+1} \frac{r-r_{m}}{r_{q}-r_{m}} d r
$$

The same holds for the stiffness matrix $\mathcal{S}$ :

$$
\mathcal{S}_{p q}=\int_{-1}^{1} l_{p}(x(r)) \frac{d}{d r} l_{q}(x(r)) d r
$$

The strong formulation of the DG scheme can then be written very compactly for every cell:

$$
\frac{d}{d t} \boldsymbol{U}_{i, j}=\frac{2}{\Delta x} \mathcal{M}^{-1}\left(-\mathcal{S} \boldsymbol{F}_{i, j}+\left.\boldsymbol{F}_{i, j}\right|_{\partial \Omega_{i}}+\boldsymbol{g}_{j}\right) .
$$

The inner boundary fluxes from the second integration by parts is represented in the vector $\left.\boldsymbol{F}_{i, j}\right|_{\partial \Omega_{i}}$ and the numerical fluxes over the cell boundaries are summarized in the vector $\boldsymbol{g}_{j}$. The first entry contains the flux of the $j$-th variable between cell $i-1$ and $i$ and the last entry the numerical flux between $i$ and $i+1$. The other entries are zero. Note that the mass matrix is in $\mathcal{M} \in \mathbb{R}^{(p+1)^{2}}$ and thus can be inverted with reasonable computational cost.

\subsection{Limiter}

Numerical schemes with order higher than one in the spatial discretization produce unphysical oscillations at steep gradients and discontinuities in the solution. These must be eliminated or at least controlled, because they might cause breakdowns in the computation (e.g. negative densities). The solution to this problem is to introduce limiters to make the numerical solution total variation bounded (TVB). This is done for every variable separately and therefore the subscript $j$ is dropped in this section.

We will make use of the modified minmod function. In its general form this is

$$
\bar{m}\left(a_{1}, \ldots, a_{m}\right):=\operatorname{minmod}\left(a_{1}, a_{2}+M \Delta x \operatorname{sign}\left(a_{2}\right), \ldots, a_{m}+M \Delta x^{2} \operatorname{sign}\left(a_{m}\right)\right) .
$$


The number $M \geq 0$ depends on the problem and is not known a-priori. If $M$ is chosen too small, the local dissipation and order reduction are increased. If, on the other hand, $M$ is chosen too large, oscillations occur [8].

The first step in the limiting procedure is to look for cells in which oscillations might occur and mark them as so-called troubled cells. The high order accuracy of the DG method should not be limited in smooth regions of the solution. This is done with the generalized limiter

$$
\begin{aligned}
& U_{i}^{\lim , L}=\bar{U}_{i}-\operatorname{minmod}\left(\bar{U}_{i}-U_{i,, 1}, \bar{U}_{i+1}-\bar{U}_{i}, \bar{U}_{i}-\bar{U}_{i-1}\right), \\
& U_{i}^{\lim , R}=\bar{U}_{i}+\operatorname{minmod}\left(U_{i, \cdot, p+1}-\bar{U}_{i}, \bar{U}_{i}-\bar{U}_{i+1}, \bar{U}_{i-1}-\bar{U}_{i}\right) .
\end{aligned}
$$

Then, if $U_{i}^{\lim , L}=U_{i,, 1}$ and $U_{i}^{\lim , R}=U_{i, \cdot, p+1}$ the cell is not marked as a troubled cell. In this case, all three input arguments of the minmod function have the same sign and the jump from the average solution in cell $i$ to its boundary value has the smallest absolute value.

The second step is the actual limiting of the troubled cells. The problem that the limiter destroys high order accuracy in regions of smooth extrema remains present, since local extrema are detected as oscillations. In order to improve this, we limit the solution with a so-called modified minmod limiter. We use the Monotone Upstream-centered Scheme for Conservation Laws (MUSCL) limiter [8]

$$
\Pi U_{i}(x)=\bar{U}_{i}+\left(x-x_{i, c}\right) \operatorname{minmod}\left(\left(U_{i}\right)_{x}, \frac{\bar{U}_{i+1}-\bar{U}_{i}}{\Delta x}, \frac{\bar{U}_{i}-\bar{U}_{i-1}}{\Delta x}\right) .
$$

Using the limited solution $\Pi U_{i}(x)$ at every numerical time integration step guarantees a total variation bounded solution.

\subsection{Fluid boundaries}

Boundary conditions for the Discontinuous Galerkin scheme are modeled with so-called ghost cells. These are artificial cells at the ends of the computational domain $\Omega$. The left end of the tube $\left(x_{\mathrm{LB}}\right)$ does not move. Static reflective boundary conditions are used to model this. For the acoustic equations, this is modeled with the ghost cell state values

$$
\boldsymbol{U}_{0}^{\mathrm{ac}}=\left(\begin{array}{c}
\rho_{1} \\
-\bar{\rho} u_{1}
\end{array}\right)
$$

The left boundary treatment for the Euler equations is correctly modeled with the ghost cell values

$$
\rho_{0}=\rho_{1}, \quad u_{0}=-u_{1}, \quad p_{0}=p_{1},
$$

respectively for the conservative variables

$$
\boldsymbol{U}_{0}^{\text {Euler }}=\left(\begin{array}{c}
\rho_{1} \\
-\rho_{1} u_{1} \\
E_{1}
\end{array}\right)
$$

It can be shown that solving a Riemann problem between the left inner cell of the domain and the ghost cell gives exactly the desired zero velocity [9].

The right end of the tube is defined by the position of the piston. Therefore, a boundary condition for moving reflective boundaries must be introduced. We interpret this as the limit of 
a subsonic outflow boundary condition. This reads [9]

$$
\boldsymbol{U}_{N+1}^{\mathrm{ac}}=\left(\begin{array}{c}
\rho_{N} \\
-\bar{\rho} u_{N}+2 \rho_{N} u_{\text {wall }}
\end{array}\right), \quad \boldsymbol{U}_{N+1}^{\text {Euler }}=\left(\begin{array}{c}
\rho_{N} \\
-(\rho u)_{N}+2 \rho_{N} u_{\text {wall }} \\
E_{N}
\end{array}\right) .
$$

The computational mesh is not changed, so at some point, the moving wall will reach a new cell. If a new cell has to be added, it gets the initial values

$$
\boldsymbol{U}_{\text {new }}^{\mathrm{ac}}=\left(\begin{array}{c}
\rho_{N} \\
\bar{\rho} u_{\text {wall }}
\end{array}\right), \quad \boldsymbol{U}_{\text {new }}^{\text {Euler }}=\left(\begin{array}{c}
\rho_{\star} \\
\rho_{\star} u_{\star} \\
\frac{1}{2} \rho_{\star} u_{\star}^{2}+\frac{p_{\star}}{(\gamma-1) \rho_{\star}}
\end{array}\right)
$$

for the acoustic equations and the Euler equations, respectively. The star values are the exact solution values of the boundary Riemann problem defined in [9].

\subsection{Monolithic formulation}

In this section, we introduce the monolithic formulation for the piston problem using the acoustic equations. In its general semi-discrete formulation it has the structure

$$
\frac{d}{d t} \boldsymbol{X}=\boldsymbol{M} \boldsymbol{X}+\boldsymbol{F}_{\mathrm{ext}}
$$

with the vector of unknowns

$$
\boldsymbol{X}=\left(\begin{array}{l}
\boldsymbol{U} \\
\boldsymbol{V}
\end{array}\right)
$$

$\boldsymbol{U}$ represents the fluid variables and $\boldsymbol{V}$ the spring variables. $\boldsymbol{M}$ is the system matrix. The system matrix is a block matrix describing the fluid and the spring discretization as well as the coupling between the two systems.

First of all, we need to introduce some general matrices. We write the identity matrix as $\boldsymbol{I}^{N}$. The superscript indicates the dimension as $N \times N$. The matrices $\boldsymbol{I}_{-1}^{N}$ and $\boldsymbol{I}_{+1}^{N}$ have ones on the sub- and superdiagonal respectively. $\left.\boldsymbol{I}^{p+1}\right|_{\partial \Omega_{i}}$ has the entries -1 in the top left corner and 1 in the bottom right corner. Finally, we use the Kronecker product $\otimes$ to define the matrices

$$
\begin{array}{ll}
\boldsymbol{I}_{11}=\left(\begin{array}{llll}
0 & \cdots & 0 & 1
\end{array}\right) \otimes\left(\begin{array}{c}
1 \\
0 \\
\vdots \\
0
\end{array}\right), & \boldsymbol{I}_{12}=\left(\begin{array}{cccc}
1 & 0 & \cdots & 0
\end{array}\right) \otimes\left(\begin{array}{c}
1 \\
0 \\
\vdots \\
0
\end{array}\right), \\
\boldsymbol{I}_{21}=\left(\begin{array}{llll}
0 & \cdots & 0 & 1
\end{array}\right) \otimes\left(\begin{array}{c}
0 \\
\vdots \\
0 \\
1
\end{array}\right), & \boldsymbol{I}_{22}=\left(\begin{array}{llll}
1 & 0 & \cdots & 0
\end{array}\right) \otimes\left(\begin{array}{c}
0 \\
\vdots \\
0 \\
1
\end{array}\right) .
\end{array}
$$


The DG discretization for the single cells is defined with the following matrices:

$$
\begin{aligned}
\boldsymbol{A}_{c} & =\frac{2}{\Delta x}\left(\boldsymbol{I}^{d} \otimes \mathcal{M}^{-1}\right)\left[-\left(\boldsymbol{I}^{d} \otimes \mathcal{S}\right)\left(\boldsymbol{F} \otimes \boldsymbol{I}^{p+1}\right)+\left(\left.\boldsymbol{F} \otimes \boldsymbol{I}^{p+1}\right|_{\partial \Omega_{i}}\right)\right], \\
\boldsymbol{G}_{l+} & =\frac{2}{\Delta x}\left(\boldsymbol{I}^{d} \otimes \mathcal{M}^{-1}\right)\left[\boldsymbol{F}^{+} \otimes \boldsymbol{I}_{11}\right], \\
\boldsymbol{G}_{l-} & =\frac{2}{\Delta x}\left(\boldsymbol{I}^{d} \otimes \mathcal{M}^{-1}\right)\left[\boldsymbol{F}^{-} \otimes \boldsymbol{I}_{12}\right], \\
\boldsymbol{G}_{r+} & =\frac{2}{\Delta x}\left(\boldsymbol{I}^{d} \otimes \mathcal{M}^{-1}\right)\left[\boldsymbol{F}^{+} \otimes \boldsymbol{I}_{21}\right], \\
\boldsymbol{G}_{r-} & =\frac{2}{\Delta x}\left(\boldsymbol{I}^{d} \otimes \mathcal{M}^{-1}\right)\left[\boldsymbol{F}^{-} \otimes \boldsymbol{I}_{22}\right] .
\end{aligned}
$$

Together, they define the matrix for the fluid flow discretization. However, the boundary treatment is not included yet:

$$
\begin{aligned}
\boldsymbol{A} & :=\left(\begin{array}{ccccc}
\left.\boldsymbol{I}^{N} \otimes \boldsymbol{A}_{c}\right)+\left(\boldsymbol{I}_{-1}^{N} \otimes \boldsymbol{G}_{l+}\right)+\left(\boldsymbol{I}^{N} \otimes \boldsymbol{G}_{l-}\right)-\left(\boldsymbol{I}^{N} \otimes \boldsymbol{G}_{r+}\right)-\left(\boldsymbol{I}_{+1}^{N} \otimes \boldsymbol{G}_{r-}\right) \\
= \\
\boldsymbol{A}_{c}+\boldsymbol{G}_{l-}-\boldsymbol{G}_{r+} & -\boldsymbol{G}_{r-} & -\boldsymbol{G}_{r-} & \\
\boldsymbol{G}_{l+} & \boldsymbol{A}_{c}+\boldsymbol{G}_{l-}-\boldsymbol{G}_{r+} & \boldsymbol{A}_{c}+\boldsymbol{G}_{l-}-\boldsymbol{G}_{r+} & -\boldsymbol{G}_{r-} \\
& \boldsymbol{G}_{l+} & \ddots & \ddots & \ddots
\end{array}\right) .
\end{aligned}
$$

The static boundary at the left end, as described in (33), becomes the matrix

$$
\boldsymbol{G}_{\mathrm{LB}}:=\left(\begin{array}{cccc}
1 & 0 & \cdots & 0 \\
0 & \cdots & \cdots & 0 \\
\vdots & & & \vdots \\
0 & \cdots & \cdots & 0
\end{array}\right) \otimes\left[\frac{2}{\Delta x}\left(\boldsymbol{I}^{d} \otimes \mathcal{M}^{-1}\right)\left[\left(\begin{array}{cc}
\frac{c}{2} & -\frac{1}{2} \\
\frac{c^{2}}{2} & -\frac{c}{2}
\end{array}\right) \otimes \boldsymbol{I}_{12}\right]\right] .
$$

The numerical modeling for the right, movable wall was described in (36). It is split into two parts. The first one is

$$
\boldsymbol{G}_{\mathrm{RB}}:=\left(\begin{array}{cccc}
0 & \cdots & \cdots & 0 \\
\vdots & & & \vdots \\
0 & \cdots & \cdots & 0 \\
0 & \cdots & 0 & 1
\end{array}\right) \otimes\left[\frac{2}{\Delta x}\left(\boldsymbol{I}^{d} \otimes \mathcal{M}^{-1}\right)\left[\left(\begin{array}{cc}
-\frac{c}{2} & -\frac{1}{2} \\
\frac{c^{2}}{2} & \frac{c}{2}
\end{array}\right) \otimes \boldsymbol{I}_{21}\right]\right]
$$

and models the reflections that are independent of the spring. The second part models the coupling from the spring to the fluid. It is

$$
\boldsymbol{B}=\left(\begin{array}{c}
0 \\
\vdots \\
0 \\
1
\end{array}\right) \otimes\left[\left(\boldsymbol{I}^{d} \otimes \frac{2}{\Delta x} \mathcal{M}^{-1}\right)\left(\begin{array}{cc}
0 & 0 \\
\vdots & \vdots \\
0 & 0 \\
0 & -\bar{\rho} \\
0 & 0 \\
\vdots & \vdots \\
0 & 0 \\
0 & c \bar{\rho}
\end{array}\right)\right]
$$


The matrix for the spring is defined as

$$
\boldsymbol{D}:=\left(\begin{array}{cc}
0 & 1 \\
-\frac{k}{m} & 0
\end{array}\right)
$$

The coupling from the fluid to the spring is done with the matrix $C \in \mathbb{R}^{2 \times N d(p+1)}$. Remember that $\rho$ actually is a disturbance in the mean density and is denoted as $\tilde{\rho}$. For the coupling, this must be taken into account and the contribution of the mean density has to be added. The total contribution must be $\frac{1}{m} c^{2} \rho_{N, p+1}+\frac{1}{m} c^{2} \bar{\rho}$. Thus, the coupling matrix is

$$
\boldsymbol{C}:=\left(\begin{array}{ccccccc}
0 & \cdots & \cdots & \cdots & \cdots & \cdots & 0 \\
0 & \cdots & 0 & \frac{c^{2}}{m} & 0 & \cdots & 0
\end{array}\right),
$$

where the non-zero entry is in row 2 and in column $N d(p+1)-(p+1)$. The contribution of the mean density goes into the term $\boldsymbol{F}_{\text {ext }} \in \mathbb{R}^{N d(p+1)+2}$. Besides this, the external pressure $p_{0}$ and the position of rest of the spring $l_{0}$ are taken into account:

$$
\boldsymbol{F}_{\mathrm{ext}}=\left(\begin{array}{c}
0 \\
\vdots \\
0 \\
-\frac{k}{m} l_{0}-\frac{1}{m} p_{0}+\frac{c^{2}}{m} \bar{\rho}
\end{array}\right) \text {. }
$$

For reasons that will become clear in the stability analysis, we introduce two correction terms $\mathcal{C}_{1}$ and $\mathcal{C}_{2}$. They account for the difference between the velocity of the piston $u_{\text {wall }}$ and the last inner velocity of the gas in the tube $u_{N, p+1}$, which introduces instabilities in the system. They are defined as

$$
\mathcal{C}_{1}=\left(\begin{array}{cccc}
0 & \cdots & \cdots & 0 \\
\vdots & & & \vdots \\
0 & \cdots & \cdots & 0 \\
0 & \cdots & 0 & 1
\end{array}\right) \otimes\left[\boldsymbol{I}^{d} \otimes \frac{2}{\Delta x} \mathcal{M}^{-1}\left[\left(\begin{array}{cc}
0 & 0 \\
0 & -c
\end{array}\right) \otimes \boldsymbol{I}_{21}\right]\right],
$$

and

$$
\mathcal{C}_{2}:=\left(\begin{array}{c}
0 \\
\vdots \\
0 \\
1
\end{array}\right) \otimes\left[\boldsymbol{I}^{d} \otimes \frac{2}{\Delta x} \mathcal{M}^{-1}\left(\begin{array}{cc}
0 & 0 \\
\vdots & \vdots \\
0 & 0 \\
0 & -c \bar{\rho}
\end{array}\right)\right]
$$

The stability analysis and a more detailed motivation for this choice of correction can be found in the next section.

We conclude with the monolithic formulation, using the above derived matrices to assemble the system matrix $M$ :

$$
\frac{d}{d t}\left(\begin{array}{l}
\boldsymbol{U} \\
\boldsymbol{V}
\end{array}\right)=\left(\begin{array}{cc}
\boldsymbol{A}+\boldsymbol{G}_{\mathrm{LB}}-\boldsymbol{G}_{\mathrm{RB}}+\mathcal{C}_{1} & \boldsymbol{B}+\mathcal{C}_{2} \\
\boldsymbol{C} & \boldsymbol{D}
\end{array}\right)\left(\begin{array}{l}
\boldsymbol{U} \\
\boldsymbol{V}
\end{array}\right)+\boldsymbol{F}_{\mathrm{ext}} .
$$

\section{STABILITY ANALYSIS}

We now want to address the subject of numerical stability, thus the response of the system to small perturbations. This is of interest, because during numerical simulations, there will always be a truncation error due to the limited computer precision. Roughly speaking, a system is stable, if small perturbations only have a small impact on the results. For the analysis we use Lyapunov's concept, which can be found in e.g. [10] in more detail. 


\subsection{Lyapunov's stability concept}

Let us first consider the ordinary differential equation

$$
\dot{\boldsymbol{x}}=\boldsymbol{f}(\boldsymbol{x}(t)), \quad \boldsymbol{x} \in \mathbb{R}^{n} .
$$

$\boldsymbol{x}_{e} \in \mathbb{R}^{n}$ is an equilibrium point of (56), if

$$
\boldsymbol{f}\left(\boldsymbol{x}_{e}\right)=\mathbf{0}, \quad \forall t \geq 0 .
$$

The equilibrium point $\boldsymbol{x}_{e}$ is said to be (Lyapunov) stable, if

$$
\forall \epsilon>0, \exists \delta>0:\left\|\boldsymbol{x}(0)-\boldsymbol{x}_{e}\right\|<\delta \Rightarrow\left\|\boldsymbol{x}(t)-\boldsymbol{x}_{e}\right\|<\epsilon, \quad \forall t>0 .
$$

Let $\boldsymbol{x}_{e}$ be an equilibrium point of (56). Further, if there exists a $C^{1}$-function $\mathcal{E}: \mathcal{U} \rightarrow \mathbb{R}$ defined on a neighborhood $\mathcal{U}$ of $\boldsymbol{x}_{e}$ with the properties

i) $\mathcal{E}\left(\boldsymbol{x}_{e}\right)=0$ and $\mathcal{E}(\boldsymbol{x})>0, \forall \boldsymbol{x} \in \mathcal{U} \backslash\left\{\boldsymbol{x}_{e}\right\}$,

ii) $\dot{\mathcal{E}}(\boldsymbol{x}(t)) \leq 0, \forall \boldsymbol{x} \in \mathcal{U} \backslash\left\{\boldsymbol{x}_{e}\right\}$,

then $\mathcal{E}(\boldsymbol{x})$ is called a Lyapunov function and the equilibrium point $\boldsymbol{x}_{e}$ is stable.

\subsection{Stability of the semi-discrete piston problem}

Lyapunov's stability concept can be simplified for the piston problem with acoustic equations. We consider the evolution of an error $\delta$ in the initial condition. Using the monolithic formulation (55), this becomes

$$
\frac{d}{d t} \operatorname{err}(t)=M \operatorname{err}(t), \quad \operatorname{err}(0)=\delta
$$

We choose the Lyapunov function candidate $\mathcal{E}(\boldsymbol{X})=\frac{1}{2} \boldsymbol{X}^{T} \boldsymbol{E} \boldsymbol{X}$ and investigate the equilibrium point $\operatorname{err}(t)=\boldsymbol{X}_{e}=\mathbf{0}$. $\boldsymbol{E}$ is called the energy matrix of the system and $\boldsymbol{X}=(\boldsymbol{U}, \boldsymbol{V})^{T}$ is the vector of unknowns. Inspired by [2] we choose the energy matrix to be

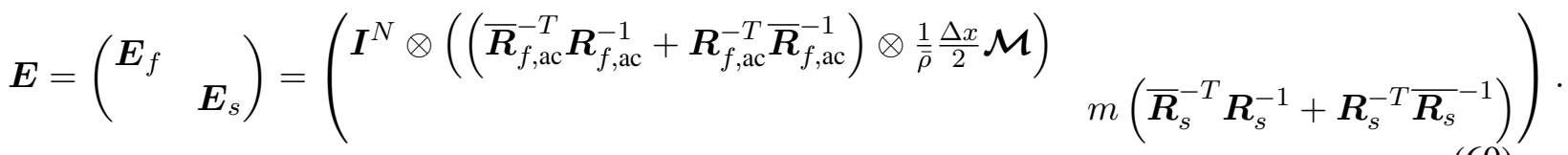

Then, $\boldsymbol{E}$ is a real symmetric positive definite matrix and the criteria $\mathcal{E}\left(\boldsymbol{X}_{e}\right)=0$ and $\mathcal{E}(X)>0$ are fulfilled. It is left to show that $\dot{\mathcal{E}}(\boldsymbol{X}(t)) \leq 0$, which turns out to consist of three subproblems:

$$
\begin{aligned}
& \boldsymbol{X}^{T} \boldsymbol{E} \boldsymbol{M}=\left(\begin{array}{l}
\boldsymbol{U} \\
\boldsymbol{V}
\end{array}\right)^{T}\left(\begin{array}{cc}
\boldsymbol{E}_{f} & \\
& \boldsymbol{E}_{s}
\end{array}\right)\left(\begin{array}{cc}
\boldsymbol{A}+\boldsymbol{G}_{L B}-\boldsymbol{G}_{R B}+\mathcal{C}_{1} & \boldsymbol{B}+\mathcal{C}_{2} \\
\boldsymbol{C} & \boldsymbol{D}
\end{array}\right)\left(\begin{array}{l}
\boldsymbol{U} \\
\boldsymbol{V}
\end{array}\right) \\
&= \underbrace{\boldsymbol{U}^{T} \boldsymbol{E}_{f} \boldsymbol{A} \boldsymbol{U}+\boldsymbol{U}^{T} \boldsymbol{E}_{f} \boldsymbol{G}_{L B} \boldsymbol{U}-\boldsymbol{U}^{T} \boldsymbol{E}_{f} \boldsymbol{G}_{R B} \boldsymbol{U}}_{1}+ \\
& \underbrace{\boldsymbol{U}^{T} \boldsymbol{E}_{f} \mathcal{C}_{1} \boldsymbol{U}+\boldsymbol{U}^{T} \boldsymbol{E}_{f} \boldsymbol{B} \boldsymbol{V}+\boldsymbol{U}^{T} \boldsymbol{E}_{f} \mathcal{C}_{2} \boldsymbol{V}+\boldsymbol{V}^{T} \boldsymbol{E}_{s} \boldsymbol{C} \boldsymbol{U}}_{2}+\underbrace{\boldsymbol{V}^{T} \boldsymbol{E}_{s} \boldsymbol{D} \boldsymbol{V}}_{3} .
\end{aligned}
$$

The single terms have the following interpretation: 
1. The DG discretization with two reflecting walls has to be stable. It can be proven that

$$
\boldsymbol{U}^{T} \boldsymbol{E}_{f} \boldsymbol{A} \boldsymbol{U}+\boldsymbol{U}^{T} \boldsymbol{E}_{f} \boldsymbol{G}_{L B} \boldsymbol{U}-\boldsymbol{U}^{T} \boldsymbol{E}_{f} \boldsymbol{G}_{R B} \boldsymbol{U} \leq 0 .
$$

2. The coupling between the fluid and the spring has to be stable:

$$
\boldsymbol{U}^{T} \boldsymbol{E}_{f} \mathcal{C}_{1} \boldsymbol{U}+\boldsymbol{U}^{T} \boldsymbol{E}_{f} \boldsymbol{B} \boldsymbol{V}+\boldsymbol{U}^{T} \boldsymbol{E}_{f} \mathcal{C}_{2} \boldsymbol{V}+\boldsymbol{V}^{T} \boldsymbol{E}_{s} \boldsymbol{C U}-\bar{\rho} c u_{N, p+1}^{2}=0
$$

The last term is a left-over from the inner DG discretization. The stability is only guaranteed with a correction.

3. The spring system has to be stable, i.e.

$$
\boldsymbol{V}^{T} \boldsymbol{E}_{s} \boldsymbol{D} \boldsymbol{V}=0
$$

In total, we get

$$
\frac{d}{d t} \mathcal{E}=\boldsymbol{X}^{T} \boldsymbol{E} \boldsymbol{M} \boldsymbol{X} \leq 0
$$

Therefore, the coupled system is (Lyapunov) stable.

Without the use of the correction terms $\mathcal{C}_{1}$ and $\mathcal{C}_{2}$, the energy analysis results in

$$
\begin{aligned}
& \boldsymbol{V}^{T} \boldsymbol{E}_{s} \boldsymbol{C} \boldsymbol{U}+\boldsymbol{U}^{T} \boldsymbol{E}_{f} \boldsymbol{B} \boldsymbol{V}-\bar{\rho} c u_{N, p+1}^{2} \\
& \quad=\rho_{N, p+1} c^{2} u_{\text {wall }}-\rho_{N, p+1} c^{2} u_{\text {wall }}+\bar{\rho} c u_{N, p+1} u_{\text {wall }}-\bar{\rho} c u_{N, p+1}^{2} \\
& \quad=\bar{\rho} c u_{N, p+1}\left(u_{\text {wall }}-u_{N, p+1}\right) .
\end{aligned}
$$

The velocity of the wall $u_{\text {wall }}$ and the velocity in the last cell $u_{N, p+1}$ are very close to each other. The difference between them will become smaller, if the spatial discretization length $\Delta x$ is reduced. Nevertheless, the difference might be positive and introduce instabilities to the system. By introducing the two correction terms $\mathcal{C}_{1}$ and $\mathcal{C}_{2}$ it is possible to eliminate this error.

\section{RESULTS}

We will compare different time integration methods for the piston problem. The Forward Euler method with time step $\Delta t$ for the monolithic formulation simply reads

$$
\boldsymbol{X}^{n+1}=\boldsymbol{X}^{n}+\Delta t \boldsymbol{M} \boldsymbol{X}^{n}+\Delta t \boldsymbol{F}_{\text {add }} .
$$

Further, we will use a Runge-Kutta 2 and a Runge-Kutta 3 method:

$$
\begin{aligned}
\boldsymbol{X}^{(1)} & =\boldsymbol{X}^{n}+\Delta t \boldsymbol{M} \boldsymbol{X}^{n}+\Delta t \boldsymbol{F}_{\mathrm{add}}, \\
\boldsymbol{X}^{n+1} & =\frac{1}{2}\left(\boldsymbol{X}^{n}+\boldsymbol{X}^{(1)}+\Delta t \boldsymbol{M} \boldsymbol{X}^{(1)}+\Delta t \boldsymbol{F}_{\mathrm{add}}\right),
\end{aligned}
$$

and

$$
\begin{aligned}
\boldsymbol{X}^{(1)} & =\boldsymbol{X}^{n}+\Delta t \boldsymbol{M} \boldsymbol{X}^{n}+\Delta t \boldsymbol{F}_{\mathrm{add}}, \\
\boldsymbol{X}^{(2)} & =\frac{1}{4}\left(3 \boldsymbol{X}^{n}+\boldsymbol{X}^{(1)}+\Delta t \boldsymbol{M} \boldsymbol{X}^{(1)}+\Delta t \boldsymbol{F}_{\mathrm{add}}\right), \\
\boldsymbol{X}^{n+1} & =\frac{1}{3}\left(\boldsymbol{X}^{n}+2 \boldsymbol{X}^{(2)}+2 \Delta t \boldsymbol{M} \boldsymbol{X}^{(2)}+2 \Delta t \boldsymbol{F}_{\mathrm{add}}\right) .
\end{aligned}
$$

Besides, we also test the Backward Euler method:

$$
\left(\boldsymbol{I}^{N d(p+1)}-\Delta t \boldsymbol{M}\right) \boldsymbol{X}^{n+1}=\boldsymbol{X}^{n}+\Delta t \boldsymbol{F}_{\text {add }} .
$$

The last time integration method tested is a simple partitioned method. It consists of two steps: 
1. The fluid system is evolved in time:

$$
\boldsymbol{U}^{n+1}=\boldsymbol{U}^{n}+\Delta t\left[\left(\boldsymbol{A}+\boldsymbol{G}_{\mathrm{LB}}-\boldsymbol{G}_{\mathrm{RB}}+\mathcal{C}_{1}\right) \boldsymbol{U}^{n}+\left(\boldsymbol{B}+\mathcal{C}_{2}\right) \boldsymbol{V}^{n}\right] .
$$

2. The spring system is evolved in time:

$$
\boldsymbol{V}^{n+1}=\boldsymbol{V}^{n}+\Delta t\left(\boldsymbol{C} \boldsymbol{U}^{n+1}+\boldsymbol{D} \boldsymbol{V}^{n}\right)+\hat{\boldsymbol{F}}_{\text {ext }} .
$$

Unless stated otherwise, a Runge-Kutta 3 method is used for the computations. We use the parameters chosen in Section 1 for the following simulations. The initial conditions are chosen to be

$$
\rho_{i, j, k}=\bar{\rho}, \quad \bar{\rho}_{i, j, k}=0 \mathrm{~kg} / \mathrm{m}^{3}, \quad x_{\text {wall }}=0 \mathrm{~m}, \quad u_{\text {wall }}=20 \mathrm{~m} / \mathrm{s} .
$$

The oscillation of the piston is depicted in Figure 3. Since there is no damping in the system, no energy should dissipate at all. This means that the oscillation amplitude should remain constant in the mean over time. It can be clearly seen that in Figure 3 the amplitude of the oscillation is conserved quite well.

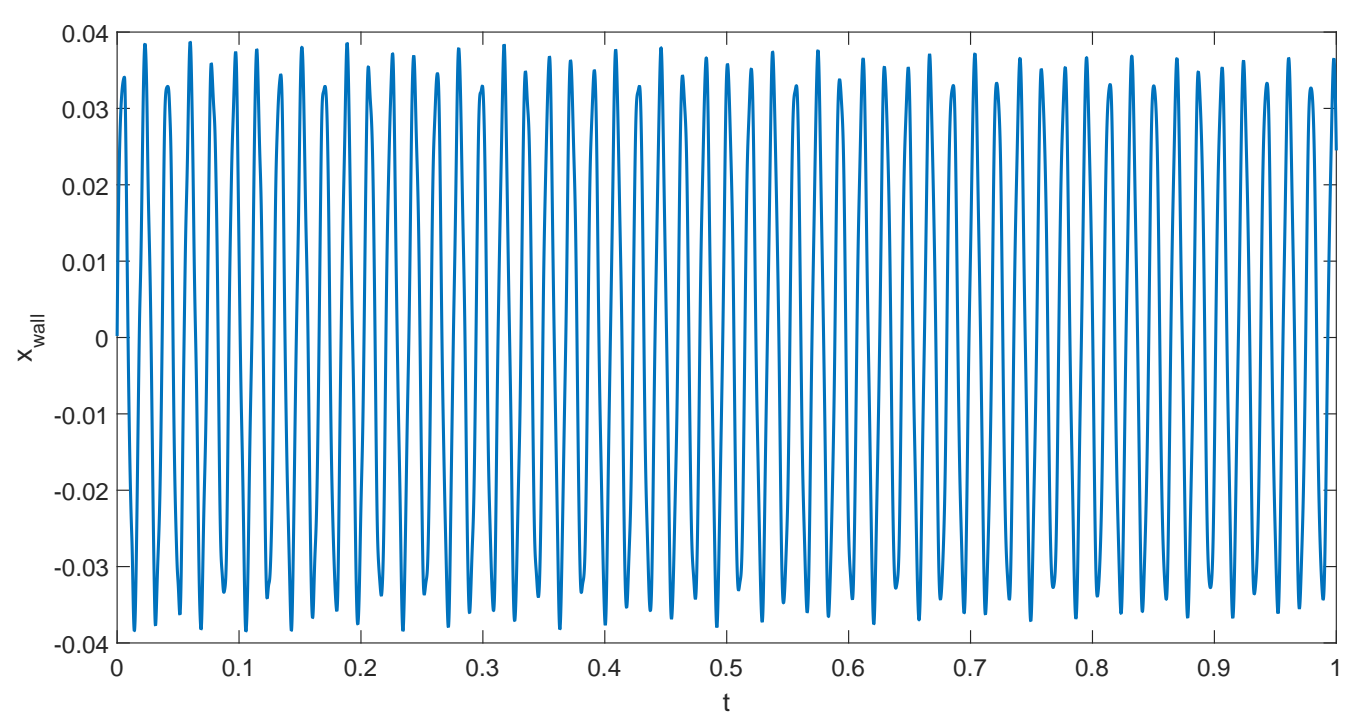

Figure 3: Position of the piston $x_{\text {wall }}$ over time.

The eigenfrequency of the simulated system is $f_{\text {sim }} \approx 54.37631 / \mathrm{s}$. This is very close to the eigenfrequency of the analytical approach in Section 1, which gives an eigenfrequency of $f \approx 54.3684^{1} / \mathrm{s}$.

The results look different when the correction terms $\mathcal{C}_{1}$ and $\mathcal{C}_{2}$ are not used. Then, the amplitude of the oscillation is increasing (Figure 4). The more cells are used, the less the impact of the instability is. Nevertheless, the amplitude keeps increasing.

In the left plot of Figure 5, the Lyapunov function of the monolithic formulation is plotted over time. The red line represents the case in which no new cells are added and removed when the piston is moving. In the other case (blue line), the computational domain is adapted to fit the piston position. Even though, the wall is moving, the domain is not adapted. This is an assumption in the analytical approach. In the right plot, the Lyapunov functions for the separated fluid (blue line) and solid (red line) parts as well as their sum (yellow line) are plotted 


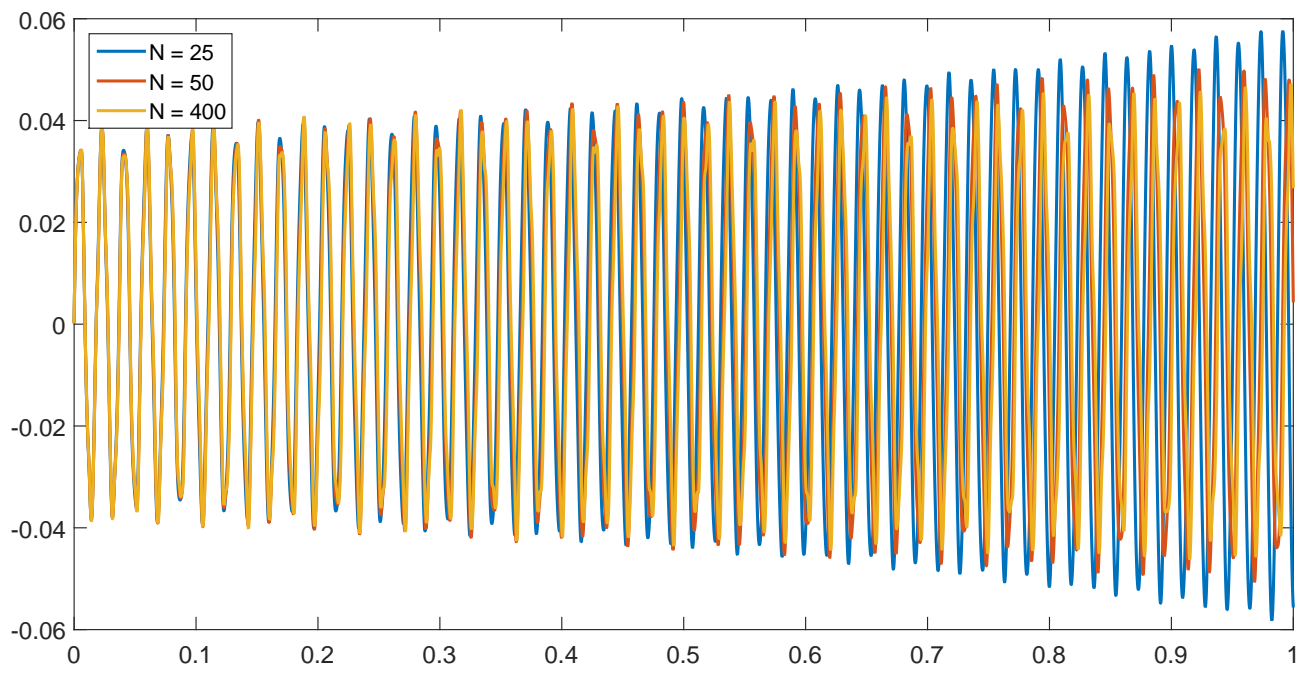

Figure 4: Position of the piston $x_{\text {wall }}$ without the use of the correction terms $\mathcal{C}_{1}$ and $\mathcal{C}_{2}$.

over time. Even though, the Lyapunov functions of the separated regions look rather irregular, their sum has a smooth shape. The decline of the Lyapunov functions over time comes from numerical dissipation during the computation and also manifests itself in a decrease of the amplitude of the piston oscillation.
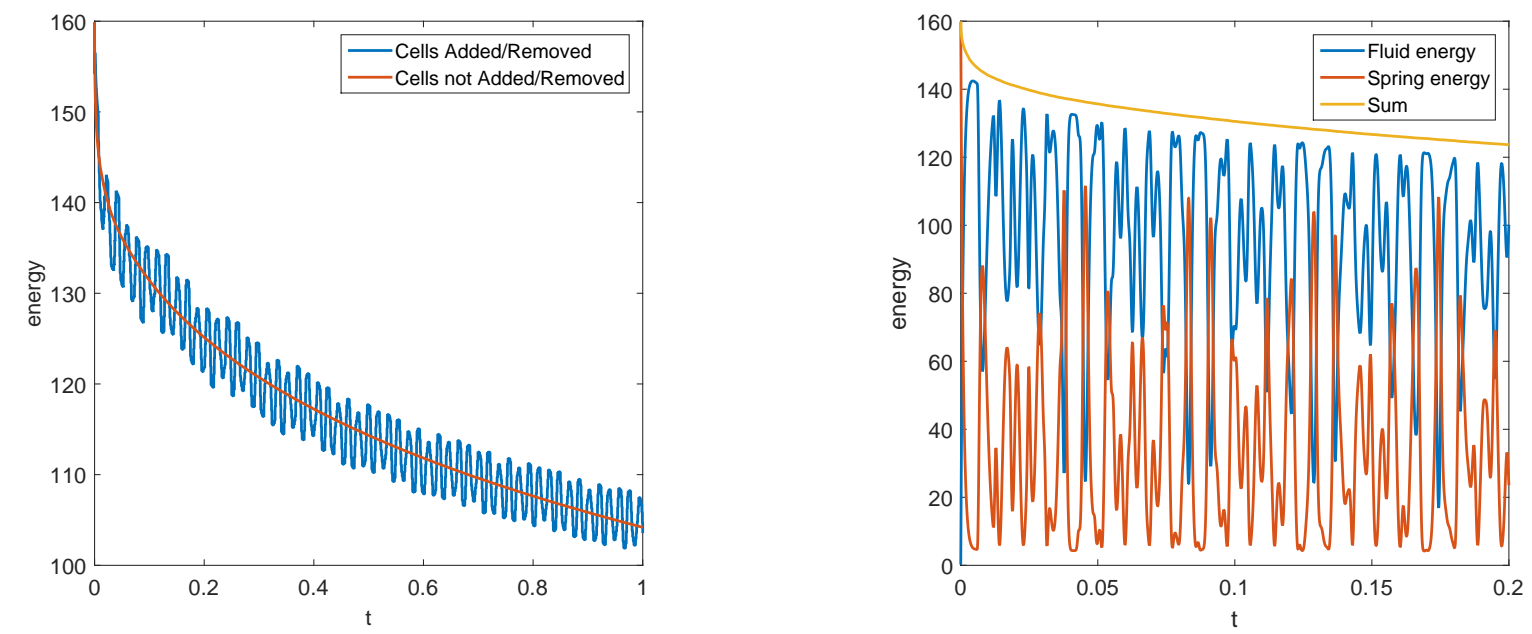

Figure 5: Left: Lyapunov functions $\mathcal{E}$ when cells are added and removed (blue line) and when the computational domain is set constant (red line). Right: Lyapunov functions for the fluid part (blue) and the structural part (red).

This (unphysical) decrease of the amplitude is small when the Discontinuous Galerkin method is used. In Figure 6 the results of a Discontinuous Galerkin method and a Finite-Volume method are compared. The Finite-Volume method can be interpreted as a Discontinuous Galerkin method with polynomial degree $p=0$. It can be seen that the higher polynomial order gives much better results as the numerical dissipation is reduced considerably.

We now want to compare the different time integration schemes introduced in the beginning of this chapter. As can be seen in Figure 7, and as expected, the simple partitioned and the Forward Euler method are unstable. The Lyapunov functions increase rapidly in the very be- 


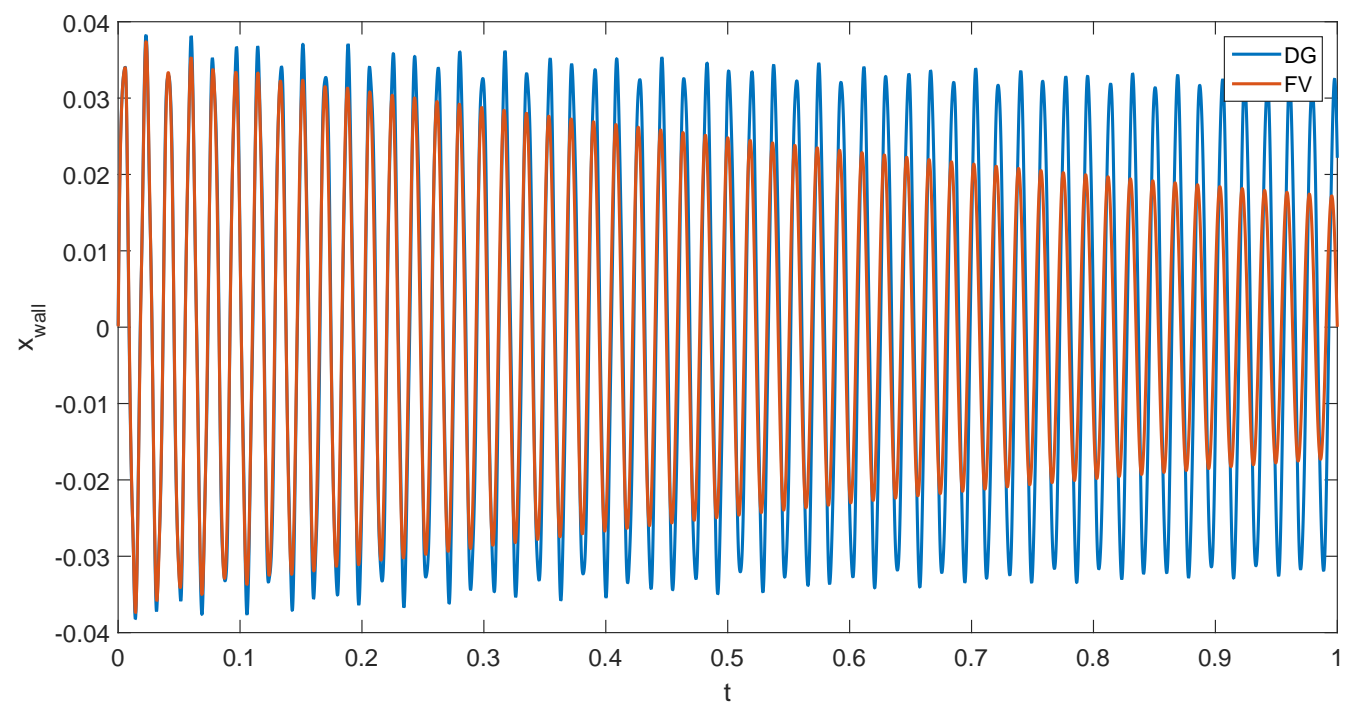

Figure 6: Position of the piston $x_{\text {wall }}$ using the Discontinuous Galerkin method ( $p=1$; blue line) and the FiniteVolume method ( $p=0$; red line).

ginning. This effect goes along with a strongly increasing amplitude of the piston oscillation. In [2] some stable partitioned methods are presented. The Backward Euler, the Runge-Kutta 2 and the Runge-Kutta 3 methods can be numerically stable. They distinguish themselves by the amount of energy that is dissipated. We see in Figure 7 that the Runge-Kutta 3 method causes the least numerical dissipation.

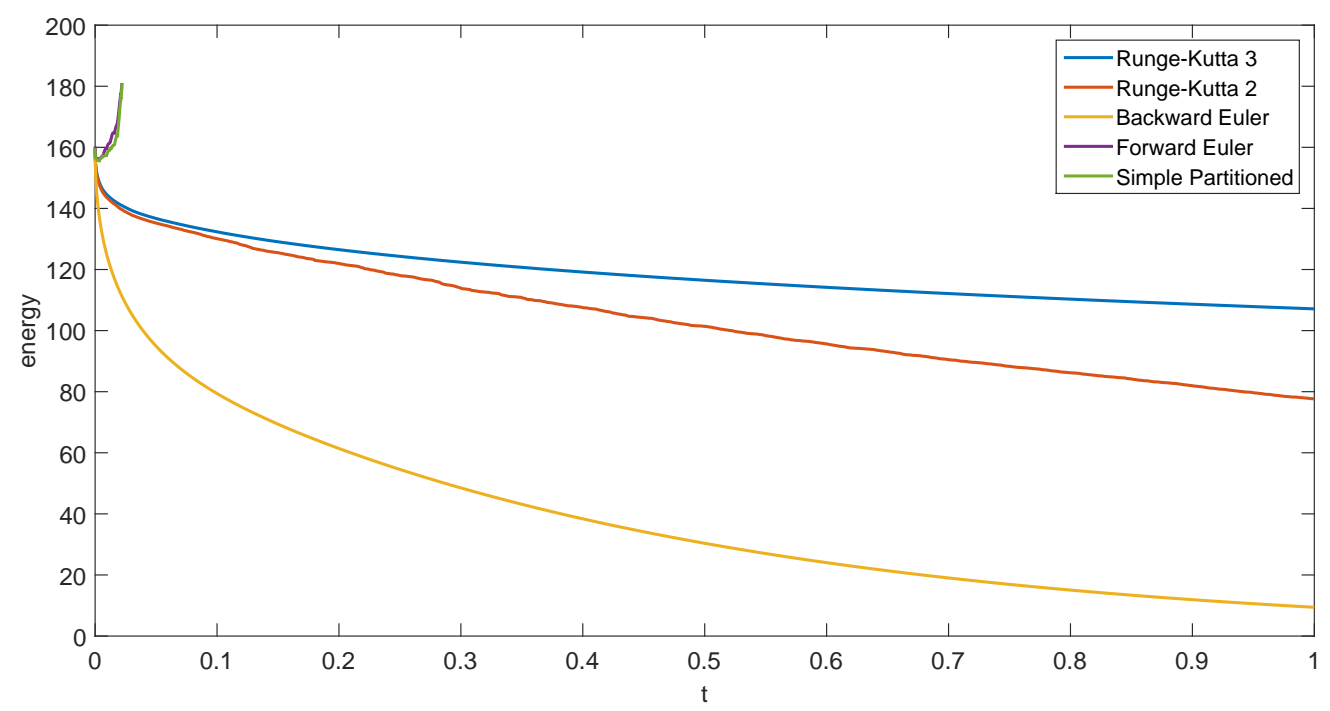

Figure 7: Lyapunov functions $\mathcal{E}$ for different time integration methods.

In the last plot, Figure 8, the oscillation of the piston is plotted over time, using the acoustic equations (blue line) and the Euler equations (red line). Especially in the beginning, the results are very much alike. 


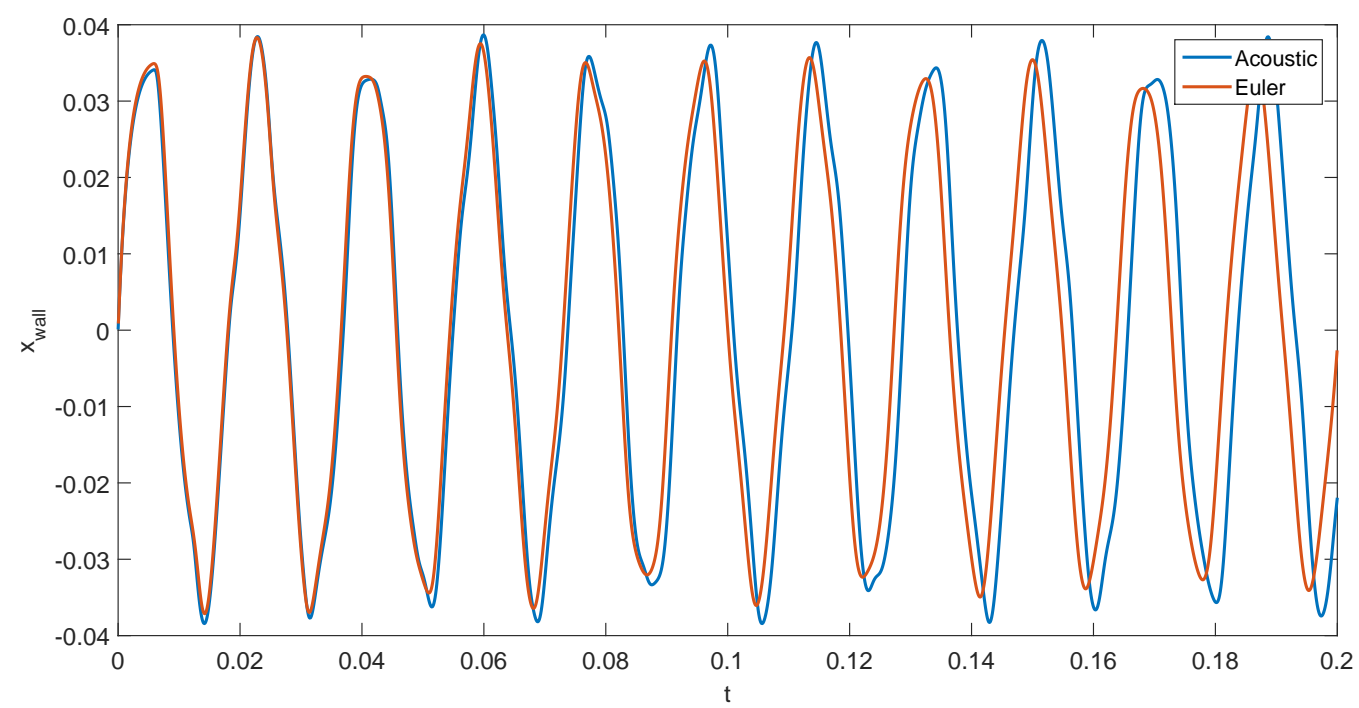

Figure 8: Position of the piston over time using the acoustic equations (blue line) and the Euler equations (red line)

\section{CONCLUSION}

We finish this work with a short summary:

- We successfully developed a monolithic simulation code for the piston problem.

- An analytical approximation of the frequency of the oscillation could be reproduced very well with our code.

- We introduced two correction terms $\mathcal{C}_{1}$ and $\mathcal{C}_{2}$ in order to achieve numerical stability for the coupling between the fluid and the structural part.

- The Discontinuous Galerkin method shows clear improvements towards the Finite-Volume method in terms of numerical dissipation.

- We tested different time integration methods on stability and numerical dissipation. In our tests, the Runge-Kutta 3 method shows the best results.

For the future, a more sophisticated boundary treatment and an extension to higher dimensions are desirable.

\section{REFERENCES}

[1] C.S. Peskin, Flow patterns around heart valves: A numerical method. Journal of Computational Physics, 10, 252-271, 1972.

[2] S. Piperno, C. Farhat, B. Larrouturou, Partitioned procedures for the transient solution of coupled aeroelastic problems - Part I: Model problem, theory and two-dimensional application. Computer Methods in Applied Mechanics and Engineering, 124, 79-112, 1995.

[3] S. Piperno, C. Farhat, Partitioned procedures for the transient solution of coupled aeroelastic problems - Part II: Energy transfer analysis and three-dimensional applications. Computer Methods in Applied Mechanics and Engineering, 190, 3147-3170, 2001. 
[4] F.J. Blom, A monolithical fluid-structure interaction algorithm applied to the piston problem. Computer Methods in Applied Mechanics and Engineering, 167, 369-391, 1998.

[5] E.H. van Brummelen, S.J. Hulshoff, R. de Borst, Energy conservation under incompatibility for fluid-structure interaction problems. Computer Methods in Applied Mechanics and Engineering, 192, 2727-2748, 2003.

[6] C. Michler, S.J. Hulshoff, E.H. van Brummelen, R. de Borst, The relevance of conservation for stability and accuracy of numerical methods for fluid-structure interaction. Computer Methods in Applied Mechanics and Engineering, 192, 4195-4215, 2003.

[7] C. Michler, S.J. Hulshoff, E.H. van Brummelen, R. de Borst, A monolithic approach to fluid-structure interaction. Computers \& Fluids, 33, 839-848, 2004.

[8] J.S. Hesthaven, T. Warburton, Nodal Discontinuous Galerkin Methods. Springer-Verlag New York, 2008.

[9] E.F. Toro, Riemann Solvers and Numerical Methods for Fluid Dynamics: A Practical Introduction, 3rd Edition. Springer-Verlag, 2009.

[10] S. Wiggins, Introduction to Applied Nonlinear Dynamical Systems and Chaos, Vol. II, 2nd Edition. Springer-Verlag New York, 2003. 